Revue d'histoire de l'Amérique française

REVUE D.HISTOIRE DE L'AMÉRIQUE FRANÇAISE

\title{
À travers le pare-brise : la création des territoires touristiques au Québec et en Ontario (1920-1945)
}

\section{Maude-Emmanuelle Lambert}

Volume 68, numéro 3-4, hiver-printemps 2015

URI : https://id.erudit.org/iderudit/1033637ar

DOI : https://doi.org/10.7202/1033637ar

Aller au sommaire du numéro

Éditeur(s)

Institut d'histoire de l’Amérique française

ISSN

0035-2357 (imprimé)

1492-1383 (numérique)

Découvrir la revue

Citer cet article

Lambert, M.-E. (2015). À travers le pare-brise : la création des territoires touristiques au Québec et en Ontario (1920-1945). Revue d'histoire de l'Amérique française, 68(3-4), 271-299. https://doi.org/10.7202/1033637ar
Résumé de l'article

Cet article lève le voile sur les différentes manières dont, historiquement, le territoire a été perçu, conçu et vécu à travers l'expérience et l'essor de la mobilité. À partir des années 1920, l'automobilité a occupé un rôle central dans la réflexion des acteurs du tourisme et dans la création des territoires touristiques. En comparant l'expérience de deux provinces, le Québec et l'Ontario, par le biais d'une analyse iconographique et discursive des guides touristiques et des récits de voyage, ce texte montre en quoi deux territoires, ayant des caractéristiques biogéophysiques semblables, développent des représentations touristiques différentes en fonction de facteurs culturels. 


\title{
À travers le pare-brise : la création des territoires touristiques au Québec et en Ontario $($ I920-1945)
}

\author{
Maude-Emmanuelle Lambert \\ Chercheure indépendante
}

RÉsumÉ - Cet article lève le voile sur les différentes manières dont, historiquement, le territoire a été perçu, conçu et vécu à travers l'expérience et l'essor de la mobilité. À partir des années 1920, l'automobilité a occupé un rôle central dans la réflexion des acteurs du tourisme et dans la création des territoires touristiques. En comparant l'expérience de deux provinces, le Québec et l'Ontario, par le biais d'une analyse iconographique et discursive des guides touristiques et des récits de voyage, ce texte montre en quoi deux territoires, ayant des caractéristiques biogéophysiques semblables, développent des représentations touristiques différentes en fonction de facteurs culturels.

ABSTRACT - This article explores the different ways in which territory has historically been perceived, conceived and practiced through the experience and growth of mobility. Since the 1920s, automobility has been seen as a major factor by the various players of the tourism industry and in the creation of touristic regions. Building on an iconographic and discursive analysis of tourist guides and travel accounts, this article compares the experience of two provinces, Quebec and Ontario, and shows how two territories with similar bio-geo-physical characteristics developed different tourist representations based on cultural factors.

1. Cet article est tiré de mes recherches doctorales : Maude-Emmanuelle Lambert, À travers le parebrise: la création des territoires touristiques à l'ère de l'automobile (Québec et Ontario, 1920-1967), thèse de doctorat (histoire), Université de Montréal, 2013, 358 p. Je remercie le Fonds de recherche du QuébecSociété et culture, le Conseil de recherche des sciences humaines du Canada, le Département d'histoire de l’Université de Montréal ainsi que le Groupe d'histoire de Montréal pour le soutien financier. L'apport intellectuel de ma directrice de recherche Michèle Dagenais est inestimable et je l'en remercie. J'aimerais également remercier les deux examinateurs externes pour leurs commentaires constructifs ainsi que Stéphane Castonguay pour m'avoir encouragée à publier mes résultats dans le cadre de ce numéro. 
$A \mathrm{u} \mathrm{XX}$ siècle, l'imaginaire du voyage en automobile a profondément les années 1910 que les premiers touristes motorisés américains franchissent la frontière. Dans l'entre-deux-guerres le nombre de ces visiteurs explose et dès 1923, ils sont 125000 touristes automobilistes à visiter le Québec, et 625000 en 1929. Les premiers gestes faits par les gouvernements québécois et ontarien en matière de développement touristique correspondent à cette affluence sans précédent. Dès lors, des liens étroits se tissent entre automobiles et mise en tourisme des territoires, et avant la fin de la Deuxième Guerre mondiale, l'automobile devient le moyen privilégié des touristes américains et canadiens pour visiter les provinces canadiennes. L'industrie touristique qui en découle se calque durablement sur le système automobile.

Au Québec, comme en Ontario, le premier ministère en charge de la question touristique est la Voirie. Créés respectivement au sein du Highways Department et du ministère de la Voirie en 1924 et 1926, le Tourist and Publicity Bureau et le Bureau provincial du tourisme ont la responsabilité de fournir de l'information touristique, de développer des infrastructures d'accueil et d'encadrer les services destinés aux visiteurs. Ce rapport entre voirie et tourisme va influencer la manière de concevoir les premiers circuits, les cartes et les guides touristiques, la publicité, voire l'hébergement. Ces éléments, qui sont autant de moyens pour transformer ou créer le territoire touristique, sont tous pensés en fonction de l'automobile 2 . C'est cette redéfinition du territoire que je souhaite ici examiner.

Comme le paysage et l'espace, le territoire est une notion polysémique, dont le sens varie en fonction des disciplines qui l'étudient et des sociétés qui le façonnent. Alors que l'espace est physique, le territoire est l'espace humanisé. Il est «conçu et perçu, approprié par des êtres humains, par un ou plusieurs peuples, et cette appropriation multiforme se déploie dans le temps ${ }^{3} »$. L’espace occupé - ou visité - n’est pas neutre: il est habité, vécu et interprété par une culture (ou plusieurs) qui nomme les choses, s'empare des lieux, les façonne et énonce ses valeurs. Le territoire se décline de différentes manières, d'abord par ses assises géographiques et

2. À ce sujet, voir Maude-Emmanuelle Lambert, «Automobile Tourism in Québec and Ontario: Development, Promotion and Representations, 1920-1945", dans Ben Bradley, Colin Coates et Jason Young, dir., Moving Natures: Environment and Mobility in Canadian History (Calgary, University of Calgary Press, à paraître).

3. Marie-Charlotte De Koninck, "Présentation. Territoire et construction identitaire», dans M.-C. De Koninck, dir., Territoires. Le Québec, habitat, ressources et imaginaire (Québec, Musée de la Civilisation et Éditions Multimondes, 2007), 1. 
politiques, par l'exploitation de ses ressources et par l'occupation de son sol, mais aussi à travers tout un imaginaire et des représentations qui lui sont propres. Le territoire est un des matériaux du paysage et le paysage, une des lectures du territoire.

Selon Gérald Domon, il y a toujours dans un paysage deux composantes essentielles. La première, le territoire, est «l'ensemble des éléments géographiques qui constituent l'assise physique du paysage, qu'ils soient d'origine anthropique (ex.: bâtiment) ou naturelle (ex.: rivière). La deuxième composante est formée par le regard qui sera porté sur ce territoire ${ }^{4}$.) Ces regards sont de natures diverses et varient en fonction des individus, des sociétés et des périodes: ils peuvent être économique, esthétique, récréatif, scientifique, agricole, écologique, environnemental ou patrimonial. En s'inspirant des travaux d'Henri Lefebvre, on pourrait dire que le territoire est à la fois le produit de perceptions ou de représentations, de conceptions ou d'organisations et de pratiques. Le perçu, le conçu et le vécu m’apparaissent donc comme les différents niveaux des transformations que connaissent les territoires par l'entremise du tourisme, tant par leur promotion et leur mise en tourisme que par les pratiques touristiques privilégiées ${ }^{5}$.

Si les liens entre la mobilité et l'expérience touristique sont bien connus, très peu de travaux se sont penchés sur la manière dont ce rapport va à son tour façonner, transformer et créer les territoires touristiques. La technologie joue ici un rôle de premier plan en créant de multiples médiations qui s'insèrent, s'intercalent et s'insinuent entre le touriste et l'environnement parcouru. Selon Marc Desportes, chaque technique de transport impose aux voyageurs des façons différentes de regarder, de considérer et de découvrir le territoire. Chacune d'entre elles modèle aussi une approche originale de l'espace traversé (qui se traduit par des pratiques d'aménagement spécifiques) et porte en soi un paysage. Ces "paysages de la technique», terme qui désigne «non pas les espaces marqués par l'omniprésence des infrastructures de transport, mais les regards induits par ces infrastructures qui les environnent», nous renseignent sur la nature complexe des liens existants entre l'homme et son milieu ${ }^{6}$.

4. Gérald Domon, «Le diagnostic paysager», dans G. Domon, dir., Le paysage humanisé au Québec. Nouveau statut, nouveau paradigme (Montréal, PUM, 2009), 139.

5. Robert S. Fieldler, "The Representational Challenge of the in-Between", dans Douglas Young, Patricia Burke Wood et Roger Keil, dir., In-Between Infrastructure: Urban Connectivity in an Age of Vulnerability (Kelowna, BC, Praxis (e)Press, 2011), 68.

6. Marc Desportes, Paysages en mouvement. Transports et perception de l'espace, XVIII-XXe siècles (Paris, Gallimard, 2005), 8. 
Des travaux récents sur l'aménagement de routes ont montré que la transformation du territoire est aussi marquée par la sensibilité des concepteurs en regard des aspects visuels et esthétiques que cette expérience devait, à leur avis, procurer aux touristes ${ }^{7}$. Différents acteurs auraient donc cherché à créer et à façonner un "regard à travers le pare-brise» (the view throught the windshield), afin de mobiliser et de satisfaire les utilisateurs. De plus, une portion importante des travaux actuels sur l'automobile s'oriente vers la prise en compte des attitudes et des comportements des utilisateurs afin, d'une part, de mieux comprendre l'appropriation et la transformation des perceptions du territoire et, d'autre part, d'expliquer les raisons de l'adoption rapide de ce moyen de locomotion.

Le concept de «motoring» défini par les historiens américains John A. Jakle et Keith A. Sculle permet d'étayer ces liens entre automobilistes et paysages. Selon ce concept, les automobilistes sont des consommateurs d'expériences de voyages "motorisés». La dimension récréative de l'automobile (sans pour autant négliger l'apport des concepteurs, de la croissance de l'industrie et du système routier) aurait donné l'impulsion à ce qui est venu par la suite, soit la motorisation du mode de vie nord-américain. L'automobile n'a pas été imposée, c'est d'abord par le plaisir qu’elle a suscité qu'elle a convaincu et séduit ${ }^{8}$. Aussi, il convient de se poser les questions suivantes: en quoi l'automobile et les dispositifs techniques qui constituent son socle influencent-ils le rapport entre l'homme et son environnement, entre le voyageur et le territoire parcouru? Et à l'inverse, comment l'idée que l'on se fait de l'environnement à partir d'une automobile en mouvement crée-t-elle et transforme-t-elle la représentation de ce territoire?

Dans le cadre de cet article, je propose d’identifier les mécanismes par lesquels se construisent les représentations du territoire en étudiant l'apport de différents acteurs, parmi lesquels figurent les gouvernements provinciaux et les touristes. Du côté des gouvernements, la création de ces représentations semble notamment s'appuyer sur un vaste travail de récupération et d'actualisation d'illustrations plus ou moins anciennes du territoire diffusées ou popularisées par l'art et de recherche dans le domaine de l'histoire et du folklore. Pour ce qui est des touristes, les

7. Voir notamment Ben Bradley, By the Road: Fordism, Automobility and Landscape Experience in the British Columbia Interior, 1920-1970, thèse de doctorat (histoire), Queen's University, 2012, 685 p.

8. John A. Jakle et Keith A. Sculle, Motoring: The Highway Experience in America (Athens, University of Georgia Press, 2008), 274 p. Voir aussi Tom McCarthy, Auto Mania: Cars, Consumers, and the Environment (New Haven, Yale University Press, 2007), 345 p. 
représentations du territoire touristique puisent abondamment dans leurs récits de voyage et leurs pratiques des territoires.

Pour documenter ces aspects, deux sources principales ont été utilisées. La première, le guide touristique, sert à mettre en valeur les attraits touristiques du territoire et à orienter le touriste dans la planification de son voyage. Pour Catherine Bertho-Lavenir, il s'avère une source polyvalente, car il permet à l'historien de voir à la fois comment s'élaborent les sites touristiques et évoluent les représentations associées à un lieu. C’est également un outil pour comprendre la manière dont les acteurs du tourisme cherchent à construire et à orienter le regard du touriste ${ }^{9}$. Certains historiens y ont toutefois vu un programme politique ${ }^{10}$. D'autres, comme Nicole Neatby, ont montré les points de convergence entre la promotion touristique et les attentes des voyageurs ${ }^{11}$. Ayant en tête ces éléments, ce travail d'analyse a porté tant sur le contenu que sur l'iconographie de ces documents. Je me suis intéressée aux couvertures, à la cartographie et à la manière dont les guides présentent le territoire (importance de la mobilité, accessibilité, etc.) ainsi qu’à la prépondérance et à la récurrence de certains thèmes et lieux ${ }^{12}$.

La deuxième source est le récit de voyage en automobile. J'ai choisi une trentaine de récits diffusés dans des revues spécialisées comme le Canadian Motorist ou publiés sous forme de monographie par des éditeurs canadiens et américains. Moins de $20 \%$ de ces témoignages sont signés par des auteurs francophones, ce qui apparaît comme un échantillon représentatif, car jusque dans les années 1950, le tourisme au Québec et en Ontario provient essentiellement du Canada anglais et des États-Unis. Plusieurs d'entre eux sont l'œuvre de journalistes ou «travelogues» (des voyageurs professionnels) comme Katherine Gordon Brinley. Certains sont aussi publiés par des auteurs primés tels que Katherine Hale (Amelia Beers Warnock de son vrai nom), une auteure originaire de l'Ontario, reconnue au Canada anglais pour sa poésie à saveur patriotique. On trouve aussi parmi ce corpus des artistes, comme les couples Hogner et Brinley, où l'un rédige et l'autre illustre l'ouvrage. Lors de la lecture de ces récits, je me suis intéressée aux milieux

9. Catherine Bertho-Lavenir, La roue et le stylo. Comment sommes-nous devenus touristes? (Paris, Odile Jacob, 1999), 438 p.

10. Rudy J. Koshar, "What Ought to be Seen," Tourist's Guidebooks and National Identities in Modern Germany and Europe", Journal of Contemporary History, 33, 3 (1998): 323-340.

11. Nicole Neatby, «Meeting of Minds: North American Travel Writers and Government Tourist Publicity in Quebec, 1920-1955", Histoire sociale, 36, 72 (novembre 2003): 465-495.

12. Dans le traitement de mes sources, j'ai abordé les images comme des constructions qu'il convient d'analyser au même titre que n'importe quel document textuel. Voir notamment Ilsen About et Clément Chéroux, «L'histoire par la photographie», Études photographiques, 10 (novembre 2001): 9-29. 
visités, aux éléments du territoire mentionnés et aux mots employés pour les décrire. Je présenterai d'abord les milieux mis en valeur par chacune des provinces dans leur promotion touristique respective, puis les récits des automobilistes en relevant les différentes manières dont ils désignent le territoire.

\section{PROMOUVOIR LE TERRITOIRE TOURISTIQUE}

Les premiers guides publiés par les gouvernements ontarien et québécois s'adressent à un public automobiliste en provenance des États-Unis. Ils cherchent avant tout à projeter l'image d'un territoire propice à la mobilité. Les couvertures des guides québécois mettent presque toujours en scène des automobilistes sillonnant les routes de Charlevoix ou de la Gaspésie, alors que les guides touristiques ontariens multiplient les images d'automobiles explorant des milieux dotés d'une géographie extrême dans l'esprit du voyageur. Des différences significatives apparaissent donc quant aux milieux naturels et aux éléments du territoire qui font l'objet d'une promotion touristique. Je présenterai, dans les deux sections suivantes, ce qui fait la spécificité de la promotion ontarienne, puis québécoise.

\section{Un Ontario sauvage et récréatif}

En Ontario, les premiers guides publiés à l'intention des automobilistes font la promotion des mêmes sites où les Torontois et les riches Américains de New York, de Pennsylvanie et d'autres États de l'Est séjournaient en villégiature au tournant du siècle. La région de Muskoka, les rives de la baie Georgienne et celles du lac Ontario maintenant accessibles par automobile, continuent d'être la marque de commerce d'un Ontario qui, fort de ses nombreux lacs, se vante d'être le plus grand terrain de jeux au pays et dans toute l'Amérique du $\operatorname{Nord}^{13}$. À la faveur de l'automobile et des développements routiers, l'Ontario étend les dimensions de son territoire touristique. Le Nord occupe une place croissante dans le paysage touristique de la province, notamment dans la promotion des premières routes à vocation récréative. Les frontières de ce Northern Country semble être continuellement repoussées. Alors qu'au tournant du $\mathrm{XX}^{\mathrm{e}}$ siècle, le Nord récréatif débute à moins d'une centaine de kilomètres au nord de Toronto (parc Algonquin, lac Simcoe, Muskoka et péninsule Bruce), au milieu des années 1930, il se situe au nord-ouest de la région torontoise, à la hauteur de Parry Sound. Ce nouveau territoire touristique comprend dorénavant

13. Beautiful Ontario, Canada's Premier Province: The Lakeland Playground of America (Toronto, Ontario, Tourist and Publicity Bureau, 1932). 


\section{Figure I}

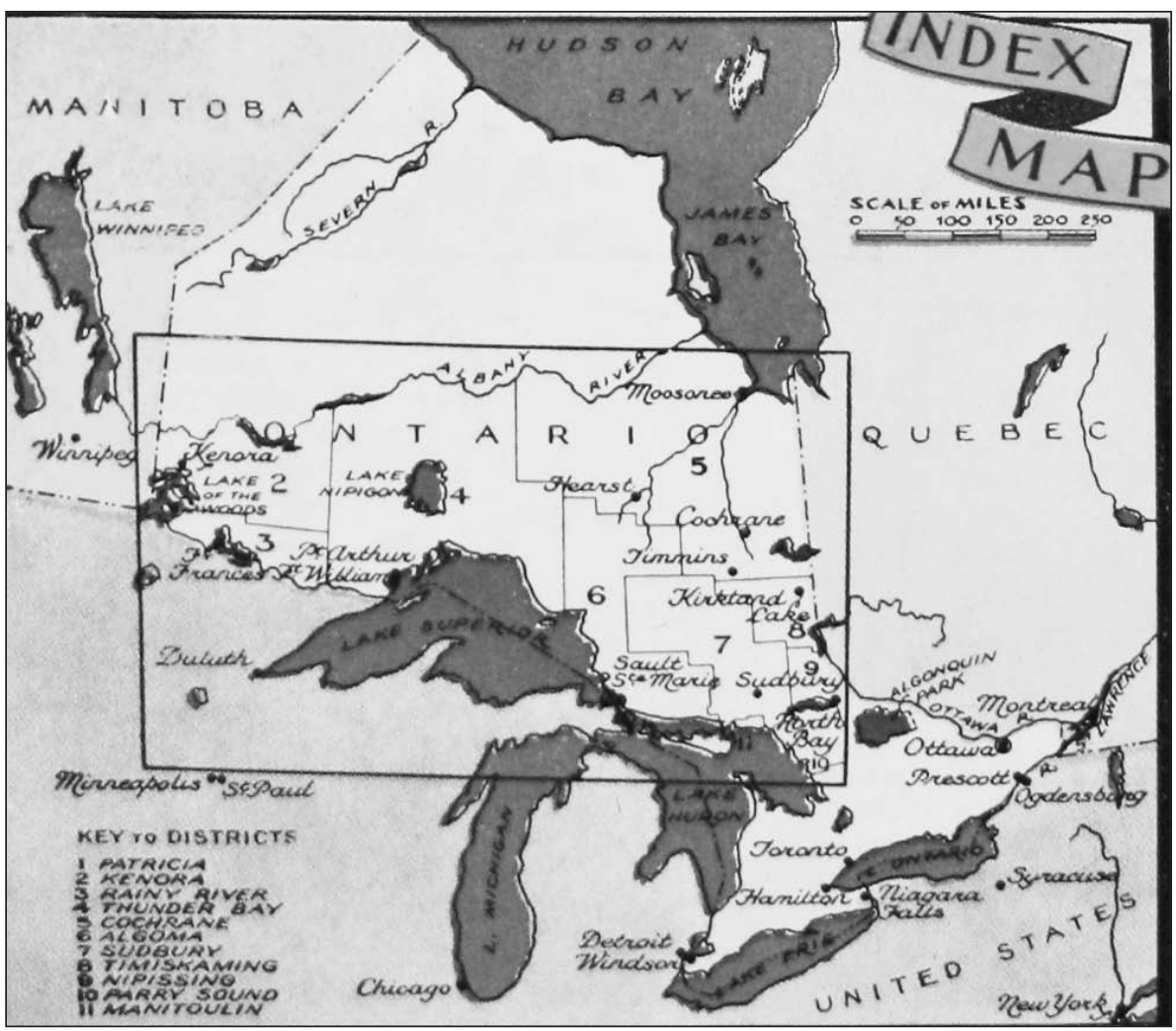

Sur cette carte, un rectangle montre les limites du Nord ontarien en tant que territoire touristique. Source: Northern Ontario, Land of Romance (1935).

la région de la baie Georgienne et tout ce qui se trouve au nord et nordouest du lac Huron et du lac Supérieur.

Lointain, mais dorénavant accessible, le Nord offre d'énormes possibilités pour ceux qui souhaitent sortir des sentiers battus. Cette publicité insiste sur la beauté et la solitude de ses paysages. Le Nord y est dépeint comme un endroit romantique, coupé de la civilisation et étroitement lié à la pratique de la chasse et de la pêche récréatives. Le guide de 1939 présente la région comme le "Canada's unspoiled fish and game country ${ }^{14}$ ». Le titre «Land of Romance» figure pendant de nombreuses années en couverture des guides de la région. Cette conception est très certainement

14. Ontario Invites You to the Lakeland Playgrounds of Canada (Toronto, Ontario Travel and Publicity Bureau, 1939). 
héritée du mouvement romantique qui a profondément marqué l'industrie touristique ontarienne depuis sa naissance au XIX ${ }^{e}$ siècle. Celle-ci a misé sur l'idée d'une tension entre la notion de civilisation et d'un état sauvage (wildness) que les milieux forestiers semblent incarner ${ }^{15}$.

En valorisant la nordicité de son territoire, l'Ontario s'inscrit aussi en porte-étendard du nationalisme canadien de l'époque. Selon l'analyse d'Eric Kaufmann, la notion de nordicité est au cœur de deux processus qui ont façonné la définition de la nation canadienne. D’une part, les Canadiens ont "nationalisé la nature», en se l'appropriant, en l'habitant, en la transformant et en l'associant historiquement à un territoire en particulier. D'autre part, ils ont "naturalisé la nation", en affirmant que la nation résulte des caractéristiques de ce territoire, qu’elle se définit par celui-ci. Source de défis et de difficultés, le Nord en vient à incarner l'essence même de la nation canadienne et permet de se distinguer, voire de se détacher de la mère patrie en se donnant une identité propre ${ }^{16}$. La construction de l'image touristique du territoire ontarien se calquerait donc en quelque sorte sur l'idée du Canada que se font les nationalistes, voire se confondrait avec la construction de l'identité canadienne-anglaise de l'entre-deux-guerres. En mettant le Nord au centre de sa promotion touristique, l'Ontario en vient à incarner l'idéal canadien, distinct à la fois d'une nature civilisée européenne et d'un Ouest américain dur et débridé.

Par conséquent, la publicité touristique de l'Ontario exploite largement une vision de la nature sauvage qui n'est pas sans rappeler celle des tableaux de Tom Thomson et des membres du Groupe des Sept. Ces derniers jouent un rôle essentiel dans la montée du nationalisme culturel canadien de l'entre-deux-guerres. En insistant sur les éléments les plus rustiques des paysages du nord du pays, le Groupe des Sept se positionne explicitement en faveur d'un art typiquement canadien ${ }^{17}$. En ayant recours aux thèmes développés par ces peintres dans sa promotion touristique, l'Ontario cherche clairement à se distinguer, mais surtout à incarner l'idéal canadien auprès des touristes canadiens et américains.

Ainsi, la ressemblance entre la couverture de l'édition de 1932 du guide officiel de la province et l'une des œuvres les plus célèbres de Thomson (The Jack Pine, 1916-1917) est évidente. La silhouette décharnée du pin,

15. Patricia Jasen, Wild Things. Nature, Culture, and Tourism in Ontario 1790-1914 (Toronto, UTP, 1995), $194 \mathrm{p}$.

16. Eric Kaufmann, "Naturalizing the Nation" : The Rise of Naturalistic Nationalism in the United States and Canada ", Comparative Studies in Society and History, 40, 4 (octobre 1998): 666-695.

17. Ibid., 685 . 
Figure 2

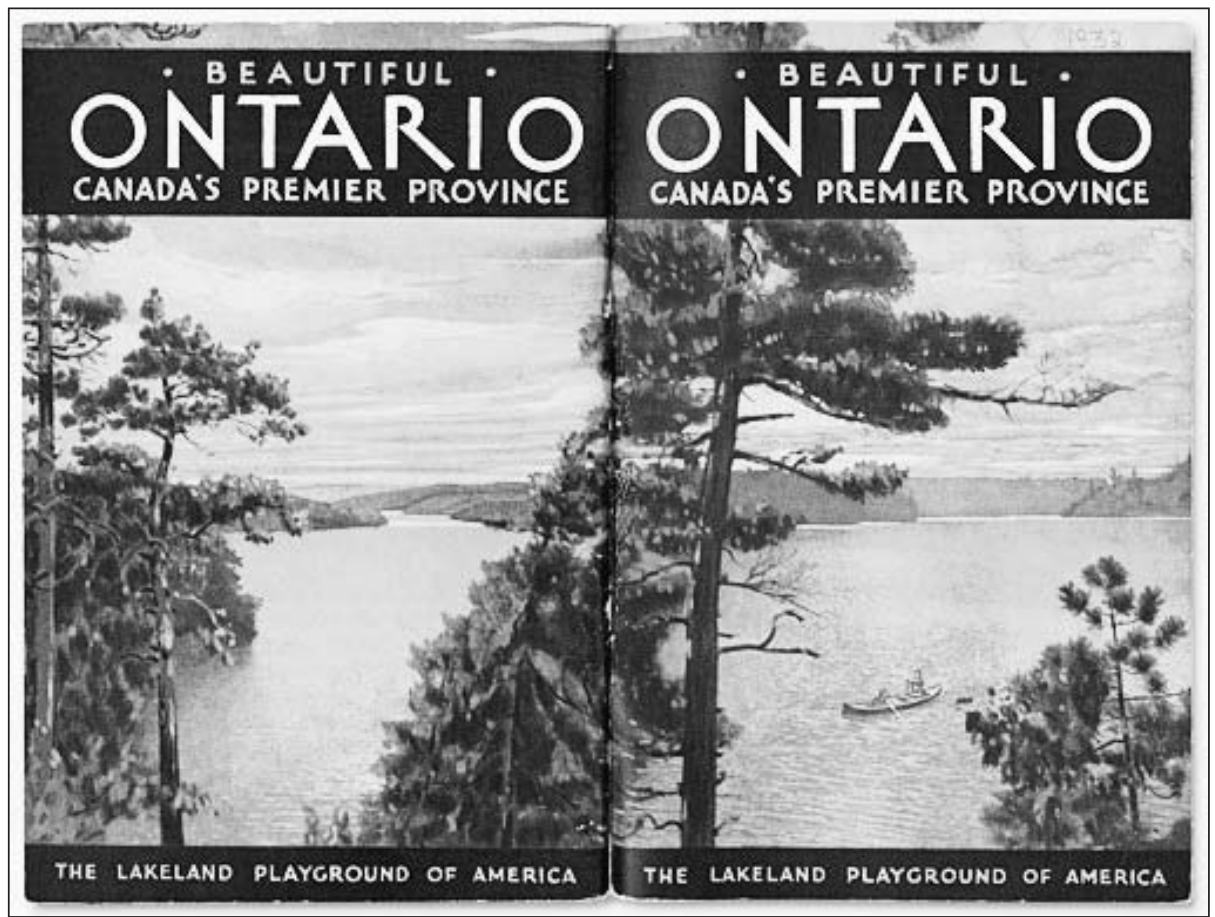

Couverture du guide Beautiful Ontario Canada's Premier Province:The Lakeland Playground of America (1932).

l'étendue d'eau et les forêts à l'horizon évoquent à la fois la rusticité et la solitude de la forêt ontarienne. Dans ces représentations artistiques, tout comme dans les guides, le Nord n'est pas un lieu habité ou lié à un travail productif, mais bien un espace récréatif ${ }^{8}$. Les populations (tant autochtones que blanches) tout comme les activités minières ou forestières, pourtant au cœur de l'économie régionale du Nord de l'Ontario, sont généralement absentes des guides touristiques. Lieu d'inspiration de Thomson, le parc Algonquin est aussi utilisé dans les guides comme figure emblématique de ce Nord à la fois récréatif et sauvage.

Toutefois, cette vision romantique de l'Ontario véhiculée par les guides ne fait pas totalement abstraction d'une présence humaine. Au contraire, les touristes sont omniprésents. Ils se livrent à différentes activités comme

18. Lynda Jessup, «The Group of Seven and the Tourist Landscape in Western Canada, or The More Things Change», Revue d'études canadiennes, 37, 1 (printemps 2002): 149. 
la chasse, la baignade, la pêche, la promenade en canot ou encore la randonnée en automobile; voilà sans doute une manière de les inciter à s'approprier ces territoires présentés comme étant «libres». La solitude n'en demeure pas moins une valeur sur laquelle la publicité touristique insiste et dans certaines images, les touristes occupent un espace minuscule par rapport au décor qui les entoure. On souligne ainsi l'aspect grandiose de la nature tout en donnant l'impression qu'ils y sont seuls. Les nombreuses photographies d'une automobile parcourant une route déserte et traversant un paysage accidenté participent à un objectif similaire.

On s'en doute, la clientèle cible de ces milieux forestiers est avant tout masculine. Toutefois, à partir des années 1930, la stratégie publicitaire de l'Ontario convoite aussi les familles, et les femmes investissent graduellement cet espace touristique. La dimension familiale du tourisme en Ontario s'affirme surtout au sud de la province, plus particulièrement dans les milieux aquatiques et dans les pratiques qui leur sont liées. La publicité vise à convaincre les familles de venir en vacances sur les bords d'un des nombreux plans d'eau de la province. Le guide de 1932 insiste sur le fait que les plages ontariennes sont parfaites et sécuritaires pour les enfants, car la descente vers les eaux est graduelle ${ }^{19}$. La nature sauvage de l'Ontario devient ici accueillante, voire sécuritaire pour un bébé.

Les rives ontariennes des lacs Érié, Huron et Ontario ainsi que la multitude de petits lacs de l'Ontario central sont au cœur de cette promotion des milieux aquatiques. On affirme même que l'automobiliste peut trouver sur presque chaque route de la province l'occasion de se jeter à l'eau. L'automobile est ainsi intégrée aux pratiques touristiques et récréatives du territoire ontarien. Dans les guides du début des années 1930, la publicité touristique incite même les vacanciers à se rendre sur les plages en automobile, voire jusqu'à l'eau. Certains y stationnent d'ailleurs leur automobile et profitent de l'occasion pour la nettoyer. D'autres s'en servent pour se protéger des regards indiscrets pendant leur baignade, comme cabines ou comme parasol.

Il faut souligner l'absence des milieux urbains et ruraux de la promotion touristique. Avant 1945, il y a très peu d'images qui leur sont consacrées et leur intérêt touristique est rarement mentionné. En fait, les scènes rurales servent à montrer aux touristes les belles routes pavées qui sillonnent la province. Par ailleurs, l'histoire, et plus particulièrement le passé 
britannique et loyaliste de l'Ontario, est l'autre grand absent de cette promotion, ce qui est d'autant plus surprenant si on considère les travaux respectifs de Cecilia Morgan et de Norman Knowles $^{20}$. Il faut rappeler que la fin du XIX ${ }^{\mathrm{e}}$ siècle et le début du XX $\mathrm{XX}^{\mathrm{e}}$ sont marqués par un besoin de se définir comme nation, d'établir ce qui fait la spécificité de la culture canadienne et plus particulièrement de l'identité canadienne-anglaise. Cette démarche identitaire qui se définit en opposition avec le voisin américain donne lieu à une volonté explicite de tisser des liens avec l'Empire ainsi qu'à de nombreuses initiatives visant à commémorer l'histoire des Loyalistes, et le passé colonial du Canada.

Entre 1934 et 1944, les différentes initiatives du ministre ontarien de la Voirie et des Travaux publics, Thomas Baker McQuesten (comme l'installation d'une série de plaques historiques commémorant l'établissement des Loyalistes et la guerre de 1812-1814) vont en ce sens. Par contre, la restauration de sites historiques comme Fort Henry, l'édification du Rainbow Bridge à Niagara Falls ainsi que la construction du Queen Elizabeth Way visaient à démontrer aux touristes américains et aux ressortissants de l'Empire britannique que l'image d'une nature sauvage propagée par le Groupe des Sept n'est pas représentative d'une nation civilisée comme le Canada. Selon l'historienne Joan Coutu, ces initiatives traduisent une vision du pays profondément ancrée dans son histoire, mais qui ont également pour objectif de promouvoir son caractère moderne et urbain ${ }^{21}$.

En somme, deux visions de la nation canadienne coexistent et sont à l'œuvre dans l'Ontario de l'entre-deux-guerres. L'une s'inscrit dans son passé colonial, tandis que l'autre s'affirme en rupture avec l'Empire. Toutefois, c'est plutôt la deuxième, celle d'une nation qui se distingue par les caractéristiques géographiques de son territoire et sa nordicité, qui est mise en valeur par les acteurs du tourisme. Pourquoi ce choix? On peut penser que la promotion d'un patrimoine culturel axé sur l'allégeance des Canadiens à l'Empire britannique, ou célébrant le passé militaire de celui-ci, n’était pas susceptible d'attirer le touriste américain, principal visiteur de la province. De plus, dans les années 1930, l'image de la nordicité canadienne est déjà familière au public américain par l'intermédiaire de la littérature, des films et de l'art.

20. Cecilia Morgan, "A Happy Holiday»: English Canadians and Transatlantic Tourism, 1870-1930 (Toronto, UTP, 2008), 416 p. ; Norman Knowles, Inventing the Loyalists: The Ontario Loyalist Tradition and the Creation of Usable Pasts (Toronto, UTP, 1997), $256 \mathrm{p}$.

21. Joan Coutu, "Vehicles of Nationalism: Defining Canada in the 1930s", Revue d'études canadiennes, 31, 1 (printemps 2002): 180-203. 
Ce besoin de se distinguer et d'affirmer son identité colore donc de manière importante la promotion touristique ontarienne. Le même processus identitaire se trouve dans la promotion touristique de l'État québécois. Toutefois, à l'inverse de l'Ontario, celui-ci met en avant essentiellement les caractéristiques agricoles de son territoire, occultant les ensembles forestiers qui pourtant prédominent largement sur son territoire à cette époque.

\section{Un Québec rural et rustique}

Les destinations touristiques traditionnelles, comme la ville de Québec et la région de Charlevoix, continuent d'occuper une position centrale dans la promotion de la province. Toutefois, au cours des années 1920 et 1930, l'État s'emploie à «vendre » aux Américains trois destinations accessibles à l'automobiliste: l'île d'Orléans, le Saguenay-Lac-Saint-Jean et surtout la Gaspésie. La publicité touristique s'adresse essentiellement au visiteur adulte et se distingue de celle de l'Ontario à la fois par l'absence des familles dans l'imagerie des guides et le type de tourisme promu. Deux clientèles principales sont visées: les amateurs de chasse et de pêche et les voyageurs motorisés attirés par un tourisme culturel. En vérité, on intensifie surtout les annonces qui concernent le deuxième type de tourisme, alors que les images de couples d'automobilistes circulant sur les routes rurales de la province abondent.

Cette valorisation de la campagne puise dans des racines profondes. Au tournant du siècle s'ouvre un vaste chantier identitaire qui amène l'élite intellectuelle à s'interroger sur ce qui forme la spécificité de la culture canadienne. Un retour aux origines s'opère alors que des chercheurs s'intéressent aux Premières Nations et au Canada français. En se penchant sur les traces matérielles de ces cultures, les ethnologues, folkloristes et historiens vont attirer l'attention sur différentes traditions, coutumes et formes artistiques ${ }^{22}$. Tout cela est récupéré par les entreprises de transport dans leur promotion d'un Québec ancien et antimoderne. Ces dernières contribuent à la publication de recueils de chansons, à l'organisation de festivals, de commémorations et d'expositions, voire au renouveau de l'artisanat. Elles axent leurs promotions sur les figures de plus en plus familières de l'Indien (dans l'Ouest canadien) et de l'Habitant (Québec), ce qui contribue à diffuser la croyance selon laquelle il existe des populations préindustrielles en marge de la société libérale moderne. Ces dernières

22. Je fais référence ici aux travaux de Marius Barbeau, Ramsay Traquair, Édouard-Zotique Massicotte ou encore Jean-Marie Gauvreau. 
Figure 3

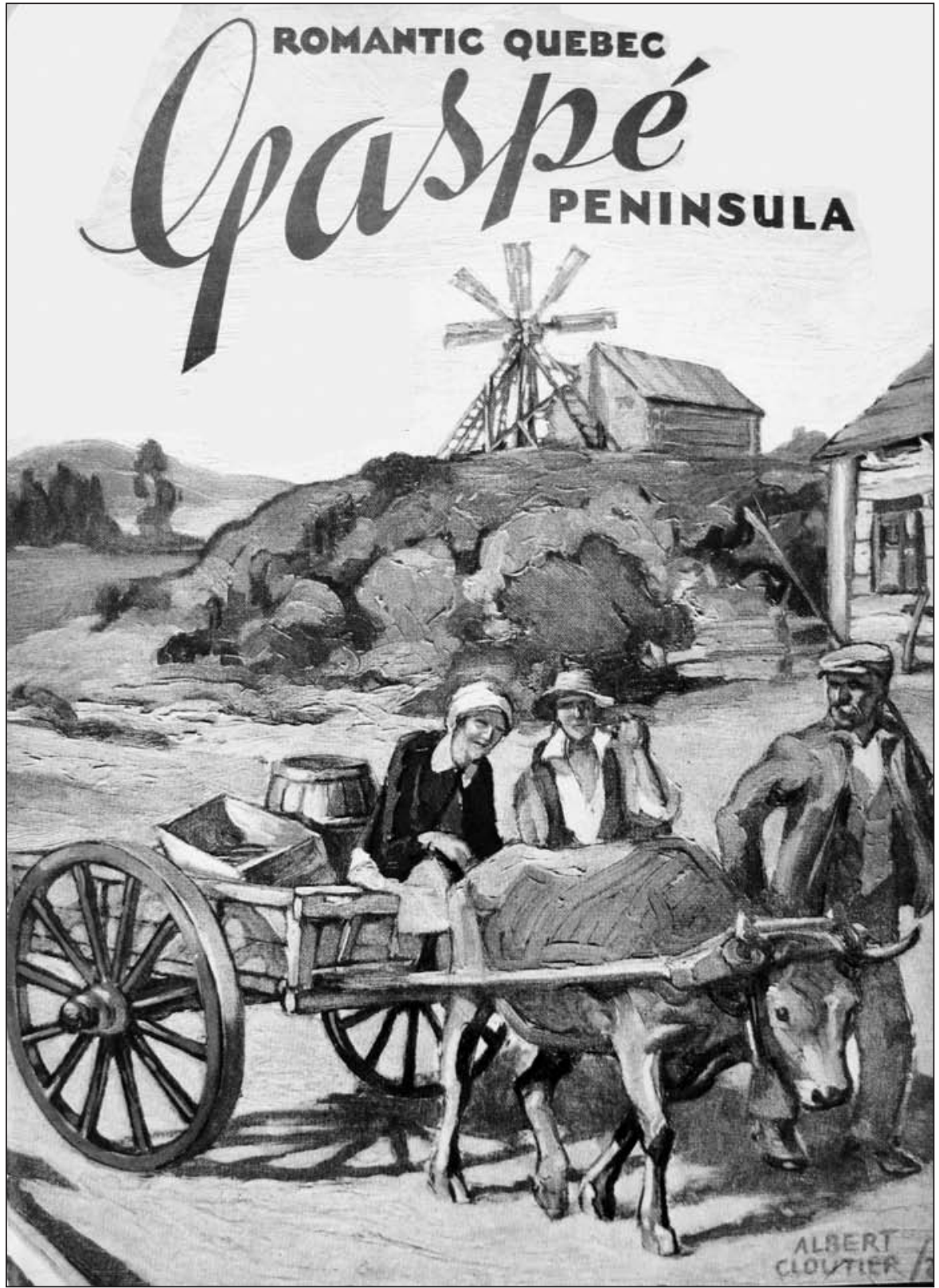

Couverture du guide Romantic Québec, Gaspe Peninsula (1935) signée par le peintre Albert Edward Cloutier, élève de A.Y. Jackson et Edwin H. Holgate. 
mèneraient "une vie simple et traditionnelle qui s'exprim[e] par des danses, des chansons, des métiers, des modes et des types d'occupation ancestrau ${ }^{23}{ }^{\prime}$. Positionnée à la périphérie de la modernité, la figure de l'habitant émerge donc comme garante de la culture essentielle à la race et à la nation.

Les premiers efforts gouvernementaux en matière de publicité touristique exploitent aussi ce filon en l'adaptant, cette fois-ci, à un public automobiliste. On fait miroiter aux touristes la possibilité d'aller en automobile à la rencontre de l'habitant et de l'observer dans sa vie quotidienne. Tout comme celle des entreprises de transport, cette publicité fait appel à des illustrateurs qui créent pour les couvertures des guides des représentations idéalisées et typiques du Canada français. Les campagnes québécoises sont présentées comme des milieux transformés par l'agriculture; une «nature cultivée » pour reprendre l'expression d'un guide de $1939^{24}$. Aussi plutôt que de postuler son caractère naturel, la promotion touristique attire l'œil de l'automobiliste sur des éléments clés d'un milieu construit et hybride, où s'imbriquent les traces d'une culture traditionnelle et d'une nature façonnée par et pour l'homme.

L'automobiliste de l'entre-deux-guerres est susceptible de rencontrer sur sa route différents marqueurs paysagers de la civilisation canadiennefrançaise et de la culture catholique. Ces éléments vernaculaires occupent une place centrale sur la couverture des guides. L'église, la maison canadienne, la croix de chemin, le four à pain extérieur et le moulin à vent sont les plus évidents et les plus récurrents. Différents éléments naturels interviennent également dans cette imagerie: des montagnes, des collines, des arbres, des plans d'eau, des terres en culture ou encore de l'herbe bien verte. Ces éléments, parfois tous présents dans la même scène, créent l'impression que la spécificité du village québécois est d'être au creux d'une vallée, entouré de montagnes, d'eau, de champs et de forêts. Cette perception s'explique par le fait que le paysage charlevoisien est régulièrement utilisé afin de donner un visage aux autres milieux ruraux ${ }^{25}$. Des espèces animales sont présentes, mais il s'agit toujours d'animaux d'élevage ou domestiqués. Alors que l'image d'un cervidé traversant une route accentue l'exotisme et le caractère sauvage de l'Ontario, bœufs et chevaux

23. James Murton, «La "Normandie du Nouveau Monde": la société Canada Steamship Lines, l'antimodernisme et la promotion du Québec ancien", RHAF, 55, 1 (été 2001): 13.

24. La Province de Québec. Pays de l'histoire, de la légende et du pittoresque (Québec, Office du tourisme de la Province de Québec, 1939).

25. Linda Villeneuve, Paysage, mythe et territorialité: Charlevoix au XIX siècle. Pour une nouvelle approche du paysage (Québec, PUL, 1999), 348 p. 
Figure 4

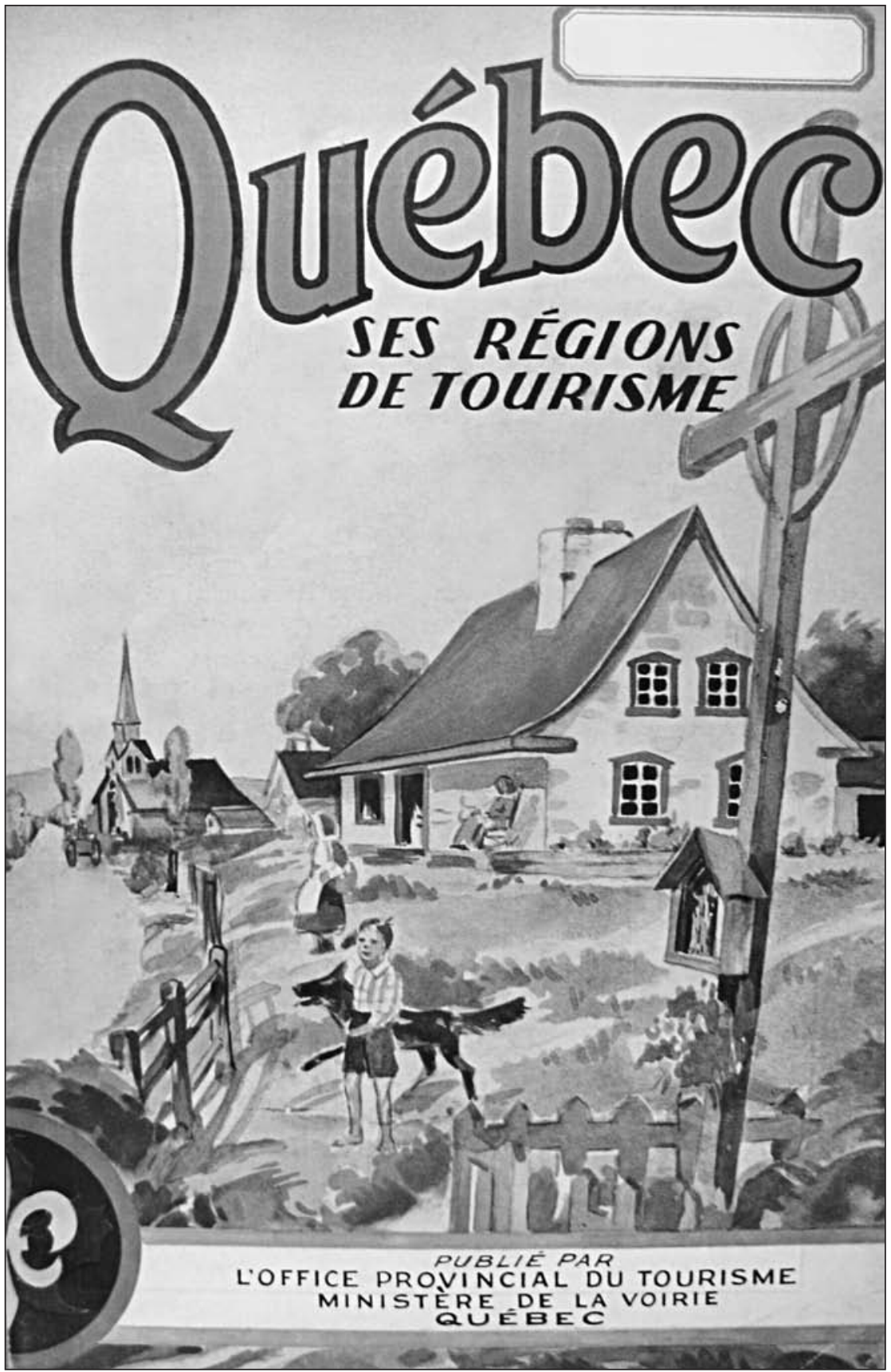

Québec, ses régions de tourisme (1934) 
sur le chemin incarnent le contraste entre la modernité de l'automobile du touriste et la vie traditionnelle des habitants du Québec.

Dans tous les cas de figure, les populations rurales sont au centre de ces représentations touristiques. Les guides suggèrent aux automobilistes qu'il est possible d'observer l'habitant dans des activités présentées comme typiques de sa vie quotidienne. Ainsi, une femme cuit son pain sur le bord de la route et un homme conduit un attelage de boufs transportant du foin. L'habitant semble aussi figé, à la fois dans l'espace et dans le temps. Hormis l'automobile et la route, le mouvement et la technologie moderne sont absents de ces images. En fait, rien ne laisse présager que les campagnes sont alors l'objet d'importants changements sociaux, culturels et économiques. Cette représentation des campagnes québécoises perdure dans l'imagerie touristique du Québec jusqu'à la Deuxième Guerre mondiale.

En fait, ces représentations des milieux ruraux correspondent à l'idée que l'étranger (l'Américain, voire le Canadien anglais) se fait de la culture canadienne-française. Comme en Ontario, on s'inspire largement du travail des artistes. Ainsi, les illustrations des guides reprennent certains des thèmes développés par les peintres du terroir: Horatio Walker (scènes de travaux agricoles de l'île d'Orléans), Clarence Gagnon (paysages charlevoisiens) et dans une certaine mesure Cornelius Krieghoff (scènes d'hiver, représentations de l'habitant et de l'Indien).

Certains points de vue tirés des œuvres de Gagnon sont même reproduits intégralement dans les dessins des couvertures de guides, comme en témoigne la figure 4 (disposition des maisons, de la route et de l'église en arrière-plan). Ces couvertures donnent l'impression d'un bricolage de différents tableaux et éléments emblématiques des milieux ruraux sur lesquels on souhaite attirer l'attention du touriste; le paysage représenté ne correspond pas à un paysage existant réellement. Des représentations du territoire se créent alors que s'opèrent un choix parmi les éléments du paysage, leur disposition et le rejet de certains aspects jugés moins désirables ${ }^{26}$.

Ces références à peine voilées aux compositions de Clarence Gagnon n'ont d'autre but que de donner le goût aux touristes de venir contempler sur place les lieux qui l'ont inspiré. Pierre-Olivier Ouellet remarque le même procédé quant à l'utilisation des œuvres d'Horatio Walker, de

26. Hélène Sicotte, Clarence Gagnon: rêver le paysage (Catalogue d'exposition, Musée national des Beaux-arts du Québec, 2006). 
Charles Huot ou encore de Cornelius Krieghoff par la Commission des Monuments historiques dans un ouvrage publié en $1928^{27}$. Le Pont de Glace (1919 ou 1920), l'un des tableaux les plus célèbres de Gagnon, figure même au verso d'un guide sur l'île d'Orléans de $1941^{28}$. Certains récits touristiques, comme celui de Jean Middleton Donald, réfèrent aux tableaux de Gagnon qui, à son avis, a su rendre les coloris des maisons ou encore ceux des paysages ruraux du Québec ${ }^{29}$.

La représentation touristique de la Gaspésie commande une promotion différente de celle du reste du Québec en raison de sa situation géographique (éloignée), de ses paysages (maritimes et montagneux) et des activités économiques (pêche et un peu d'agriculture) de sa population. Le pêcheur gaspésien est d'ailleurs un personnage qui prend une place importante au cours de la période, dans la mise en image et en récit du territoire touristique. De plus, contrairement à l'Ontario, dont on souligne à grands traits les beautés sauvages, la Gaspésie des guides touristiques est présentée de manière à accentuer son caractère rustique. Cela se traduit par la valorisation de la simplicité et du mode de vie traditionnel de sa population, jumelée à celle de la présence de la religion catholique affichée à certains endroits. Cependant, dépendamment des publics visés, la présentation de la région diffère. Ainsi, la version anglophone d'un guide de 1930 compare l'aspect physique et humain de la Gaspésie à l'Irlande, dans une référence implicite à la culture celtique ${ }^{30}$.

Le pittoresque de la région est mis en avant et le touriste est invité à ralentir afin d'avoir «[...] le temps de contempler les beautés scéniques de la région sur les différents aspects qu'elles revêtent suivant l'éclat de la lumière qui change à chaque heure du jour ${ }^{31}$ ». La Gaspésie est présentée comme un immense terrain de jeux pour l'automobiliste qui, si le désir lui en prend, peut rouler à plein régime (quoique la difficulté du parcours par endroit le rappelle vite à l'ordre). Tant en anglais qu'en français, les documents promotionnels accentuent l'antimodernité qui caractériserait la région et surtout le mode de vie des populations. Celle-ci est présentée au touriste francophone comme une manière de renouer avec ses propres

27. Pierre-Olivier Ouellet, “"Nos routes se couvrent de touristes à la recherche de nos reliques du passé”. Les débuts de la Commission des monuments historiques (1922-1928)", RHAF, 61, 2 (automne 2007): 248.

28. L'île d'Orléans (Québec, Province de Québec Tourist Bureau, 1941).

29. Jean Middleton Donald, Quebec Patchwork (Toronto, MacMillan, 1940), 186.

30. The Gaspé Peninsula, History, Legends, Resources, Attractions (Québec, Department of Highways and Mines, 1930), 10.

31. La Gaspésie: histoire, légendes, ressources, beautés (Québec, ministère de la Voirie, 1930), 8. 
racines. Il est invité à découvrir l'âme gaspésienne et à comprendre son attachement pour un sol qui lui a apporté plus de peines que de joies. Jouant en quelque sorte à l'ethnologue, l'automobiliste peut échanger avec le Gaspésien et apprécier toute la diversité de sa langue et de sa culture matérielle: «Il faut encore flâner dans les campagnes, causer avec le paysan gaspésien et l'entendre prononcer ces vieilles expressions du terroir qui tendent de plus en plus à disparaître ${ }^{32}$.»

Dans une perspective globale, l'analyse iconographique et discursive des guides met en évidence la centralité des milieux naturels dans la promotion touristique des deux provinces. Certains d'entre eux sont plus exploités que d'autres et deviennent même emblématiques. Chose certaine, l'iconographie puise abondamment dans les arts visuels et reproduit les tableaux d'artistes de renom, à qui ils empruntent leurs conceptions romantiques des milieux forestiers et ruraux. Le choix de l'art au détriment de la photographie participe ici à la construction de la représentation du territoire. La peinture permet de composer le mythe de la campagne québécoise ou celui de l'Ontario sauvage en disposant des éléments qui doivent y figurer, mais aussi en faisant abstraction de ceux qui ne doivent pas y être conformément à un certain idéal romantique. L'art contribue à entretenir ce mythe, à lui donner une patine ancienne par rapport à la photographie, alors perçue comme une technique moderne. L'art est aussi présent dans le regard porté par certains touristes sur le territoire et c'est l'une des nombreuses manières de s'approprier le territoire touristique.

\section{DÉCOUVRIR ET S'APPROPRIER LE TERRITOIRE TOURISTIQUE}

Parce qu'il est lu et diffusé, le récit touristique contribue à créer le territoire. Par l'expérience des lieux, le touriste en désigne les contours et corrobore ou remet en question le sens qui lui est attribué. Le récit permet aussi de lui donner vie, et au touriste de se l'approprier par l'entremise des pratiques, mais également de mots. Ce procédé est comparable à celui observé par nombreux historiens quant à l'appropriation culturelle d'un territoire à travers la peinture, l'aménagement paysager, la cartographie, la toponymie, les sciences naturelles, voire la pratique de la chasse récréative $^{33}$. Ici l'automobilité du touriste oriente tant ses pratiques et ses perceptions que sa façon de parler du territoire. Trois manières d'évoquer et

32. Ibid.

33. Voir Greg Gillepsie, «I Was Well Pleased with Our Sport among the Buffalo: Big-Game Hunters, Travel Writing, and Cultural Imperialism in the British North American West, 1847-1872», Canadian Historical Review, 83, 4 (décembre 2002): 555-584. 
de pratiquer le territoire se dégagent des récits, soit à travers les qualités recherchées, les paysages et la présence humaine.

\section{Les qualités du territoire touristique}

Dès les premières pages de leurs récits, les touristes présentent leurs certitudes et leurs hésitations ou encore leurs besoins d'exotisme et d'évasion. Les renseignements qu'ils puisent dans les guides orientent leur vision du Québec et de l'Ontario et contribuent à une certaine idéalisation des lieux et de ces sociétés. Toutefois, c'est au long de leur parcours que ces représentations se construisent véritablement, et parfois se déconstruisent. Aussi, est-il intéressant d'examiner quelles sont les qualités recherchées dans les territoires visités et comment celles-ci viennent se greffer ou non aux représentations existantes.

Ces qualités ne sont pas les mêmes en fonction des différentes clientèles touristiques et de la destination. Néanmoins, il existe certains points communs. Le climat est l'un d'eux. La chaleur, le froid, la pluie marquent les conditions dans lesquelles se fait le voyage en automobile et c'est pour certains Américains un des éléments qui motivent le choix d'une destination. Ainsi, les Brinley décident de fuir la chaleur estivale du Connecticut vers le «Nord». Leur voyage en automobile les mène sur les rives du SaintLaurent et de l'Outaouais, mais aussi à Montréal, à Charlevoix et au LacSaint-Jean. Dans les guides touristiques, les conditions climatiques sont toujours idéales en fonction des activités que le touriste est susceptible d'y pratiquer. Ainsi, les étés québécois sont parfaits pour voyager confortablement ${ }^{34}$. Reconnus pour leurs longues journées d'ensoleillement et pour la fraîcheur de leurs nuits, l'Ontario et le Québec sont qualifiés de revigorants pour le corps et pour l'esprit. Ainsi, l'hiver québécois est présenté comme une véritable fontaine de jouvence ${ }^{35}$, alors que la qualité de l'eau et du climat ontarien expliquerait la longévité de sa population ${ }^{36}$.

Une autre qualité de ces territoires touristiques est de favoriser l'expérience de la solitude. Explorer des territoires éloignés qui permettent de fuir et de s'isoler de la civilisation ne s'applique pas seulement à l'expérience touristique de l'Ontario. La Gaspésie est considérée comme un milieu qui offre aussi cette possibilité. Une différence majeure apparait cependant dans les récits: les touristes n'y sont pas à la recherche d'une nature sauvage. D'ailleurs, le terme "wilderness» n'est pratiquement

34. Tours in Québec, Canada, $3^{e}$ éd. (Québec, Roads Department, 1933), 3.

35. La Province de Québec, op. cit., 1.

36. Beautiful Ontario, op. cit., 28. 
jamais dans le récit, alors que l'expression "wide open space» revient constamment. La solitude n'exclut donc pas une présence humaine, mais celle-ci doit se faire rare, plus discrète. À Percé, Dorothy et Nils Hogner installent leur campement au-dessus d'une falaise de deux cents pieds de hauteur, plutôt que de camper à proximité de la ferme de l'agriculteur qui les a invités à passer la nuit chez lui. Ils choisissent ce site parce qu'il est en retrait, mais aussi parce qu'il offre une vue magnifique sur la plage et la mer $^{37}$. L'aspect accidenté du paysage et surtout le défi qu'il présente à la conduite automobile sont aussi un gage pour certains touristes américains qu'il s'agit d'un territoire peu pénétré par l'homme et la modernité. Ce paysage serait susceptible d'apporter une expérience authentique des milieux qui le composent.

Le besoin de partir, de laisser derrière soi son quotidien et ses engagements, de rompre avec un rythme de vie astreignant et planifié est une des motivations les plus communes aux touristes d'hier et d'aujourd'hui. Dans l'entre-deux-guerres, cette motivation fait souvent référence implicitement aux idées antimodernistes. Pour plusieurs automobilistes en provenance des États-Unis, camper au Québec et en Ontario se révèle être une manière authentique de pratiquer le territoire. En expérimentant un mode de vie rudimentaire, le touriste est plus à même de laisser derrière lui son quotidien et s'imagine plus apte à comprendre la relation que ses ancêtres entretenaient avec les milieux naturels. Pour les Hogner, le camping permet d'éviter les pièges touristiques et de s'éloigner des itinéraires suivis par les autres voyageurs. Ils font également un festin de petits fruits cueillis ici et là, d'un pain qu'une fermière leur a vendu ou encore d'un poisson que leur offre un pêcheur ${ }^{38}$.

Qui plus est, en cherchant à s'éloigner de la modernité, le touriste se dirige vers des milieux qu'il perçoit comme authentiques. Certains seraient donc plus propices que d'autres à favoriser ce rapport antimoderne avec la nature convoitée. Ainsi, les forêts incarnent, selon l'Américaine Kathrine Brinley, une authenticité qu'elle ne trouve nulle part ailleurs ${ }^{39}$. Du côté des touristes canadiens et plus spécifiquement québécois et ontariens dans leur province respective, la nostalgie est une motivation importante pour entreprendre un voyage. On relève souvent l'envie d'un «retour aux sources», d'explorer sa province ou encore de retrouver

37. Dorothy Childs Hogner, Summer Roads to Gaspé (New York, E. P. Dutton, 1939), 149-153.

38. D. C. Hogner, Summer Roads to Gaspé, op. cit.

39. Kathrine Gordon Brinley, Away to Quebec: A Gay Journey to the Province (New York, Dodd, Mead and Company, 1937), 9. 
un lieu que l'on a habité par le passé. Pour les voyageurs ontariens, les paysages des milieux ruraux de leur province leur rappellent un mode de vie différent de celui des villes, une façon d'exister qu'on sent sur le point de disparaître.

De leur côté, les touristes québécois ne font pas nécessairement état d'une nostalgie lorsqu'ils évoquent leur attachement aux milieux ruraux. Peu ciblés par la promotion touristique québécoise, ces derniers ne se sentent pas non plus interpellés par la possibilité de rencontrer l'habitant. Pour le touriste canadien-français, visiter la province de Québec constitue plutôt une occasion d'explorer son "foyer", sa patrie, d'accomplir un pèlerinage sur ce territoire qui regorge des vestiges d'un passé collectif ; bref, il s'agit ici d'un tourisme investi d'un lien identitaire avec le territoire visité $^{40}$. Ainsi, pour un chroniqueur de l'Automobile au Canada, l'aspect du territoire québécois et de la nature qui le compose reflète le caractère de ce peuple. Aussi, le touriste qui le parcourt sera en mesure de comprendre l'identité canadienne-française ${ }^{41}$.

Dans les récits des automobilistes ontariens qui parcourent leur province, ces liens entre nature, territoire et identité trouvent leur expression dans le rappel des ancêtres. Ainsi, Katherine Hale parle des Loyalistes venus s'installer dans la région de Kingston et de Belleville, voire des Écossais agriculteurs de l'Est ontarien. Quoique quasi absent de la promotion touristique, cet intérêt pour le passé loyaliste de la province est stimulé par les différents marqueurs qui se retrouvent sur le parcours de l'automobiliste tels que les plaques historiques installées le long du réseau routier dans les années $1930^{42}$.

Tant dans les récits sur le Québec que sur l'Ontario, l'histoire et ses traces constituent une des nombreuses qualités d'un territoire propice au tourisme. Au Québec, les figures héroïques des explorateurs, des gouverneurs et des missionnaires se mêlent à celles des coureurs des bois et des seigneurs des lieux. Dans le cas de l'Ontario, les touristes font référence aux fondateurs des villes ou aux personnages célèbres (inventeurs, écrivains, etc.) qui y sont nés. Bref, les qualités recherchées varient beaucoup plus en fonction des clientèles que des territoires. Cependant, les différents interlocuteurs, qu'ils soient francophones ou anglophones, américains ou canadiens, ont un point en commun: lorsqu'ils écrivent sur le territoire,

40. N. Neatby, «Meeting of Minds ...", loc. cit., 487.

41. Amédée Buteau, "Propos sur nos routes», L'automobile au Canada, 3, 7 (décembre 1922): 29-31.

42. N. Knowles, Inventing the Loyalists..., op. cit., 169. 
ils l'évoquent en termes de paysages qu'ils observent à partir de leur automobile en mouvement.

\section{Automobilité et paysages}

Le paysage de l'automobiliste est composé de différents éléments qui varient d'une région et d'un récit à l'autre, mais qui constituent l'assise physique du paysage visible par la fenêtre de l'automobile. Par-dessus tout, l'automobile donne lieu à une façon particulière d'évoquer les éléments qui composent le paysage. Les auteurs énumèrent les éléments qui se succèdent sur leur route à la manière de photographies. Selon le travelogue Frank Yeigh, c'est en portant attention aux petits détails que l'automobiliste pourra avoir une image précise du territoire parcouru plutôt que d'en garder un souvenir flou et confus ${ }^{43}$. Pour rendre cette impression photographique, certains auteurs utilisent des points de suspension entre les éléments du paysage observé à partir de l'automobile en mouvement:

A rolling countryside; large stone houses, big barns, square files, wind breaks of trees... men resting on the handles of their hoes, children emulating their elders... a bay horse scratching his nose against a fence post... [...] automobiles, many bearing American licences, speeding along the highway ${ }^{44}$.

Une autre façon consiste à présenter les différents éléments qui se succèdent sur la route en mode accéléré, et ce, en décrivant ses virages et ses soubresauts. L'auteur cherche ainsi à donner l'impression au lecteur qu'il fait partie de l'équipée. Kathrine Gordon Brinley mentionne les villes et villages entre Bagotville et Péribonka; au passage, elle ponctue sa description de quelques éléments sur l'histoire des lieux et leur aspect visuel. Cette narration cherche à traduire le mouvement de l'automobile et permet de rendre le récit plus dynamique, plus vivant qu’une simple énumération des éléments du paysage. Pour renforcer encore cette impression, l'auteure précise même qu'elle tente d'écrire alors que l'automobile roule:

Five miles beyond Arvida we come to Jonquière: "This town ought to be progressive," I say to Dan; "[...] Look at the attractive old houses - and new ones going up; and the path to the schoolhouse bordered by Normandy poplars. "... We run along too far before turning left and have to go back, to find the white markers of Route 16; we overtake four little children carrying berry cans in their hands ${ }^{45}$.

43. Frank Yeigh, «The Passing Panorama of a Windshield», Canadian Motorist, 17, 4 (avril 1930): 123.

44. William R. Watson, And All Your Beauty (Toronto, Macmillan Co. of Canada, 1953), 9-10.

45. K. G. Brinley, Away to Quebec..., op. cit., 90-91. 


\section{Figure 5}

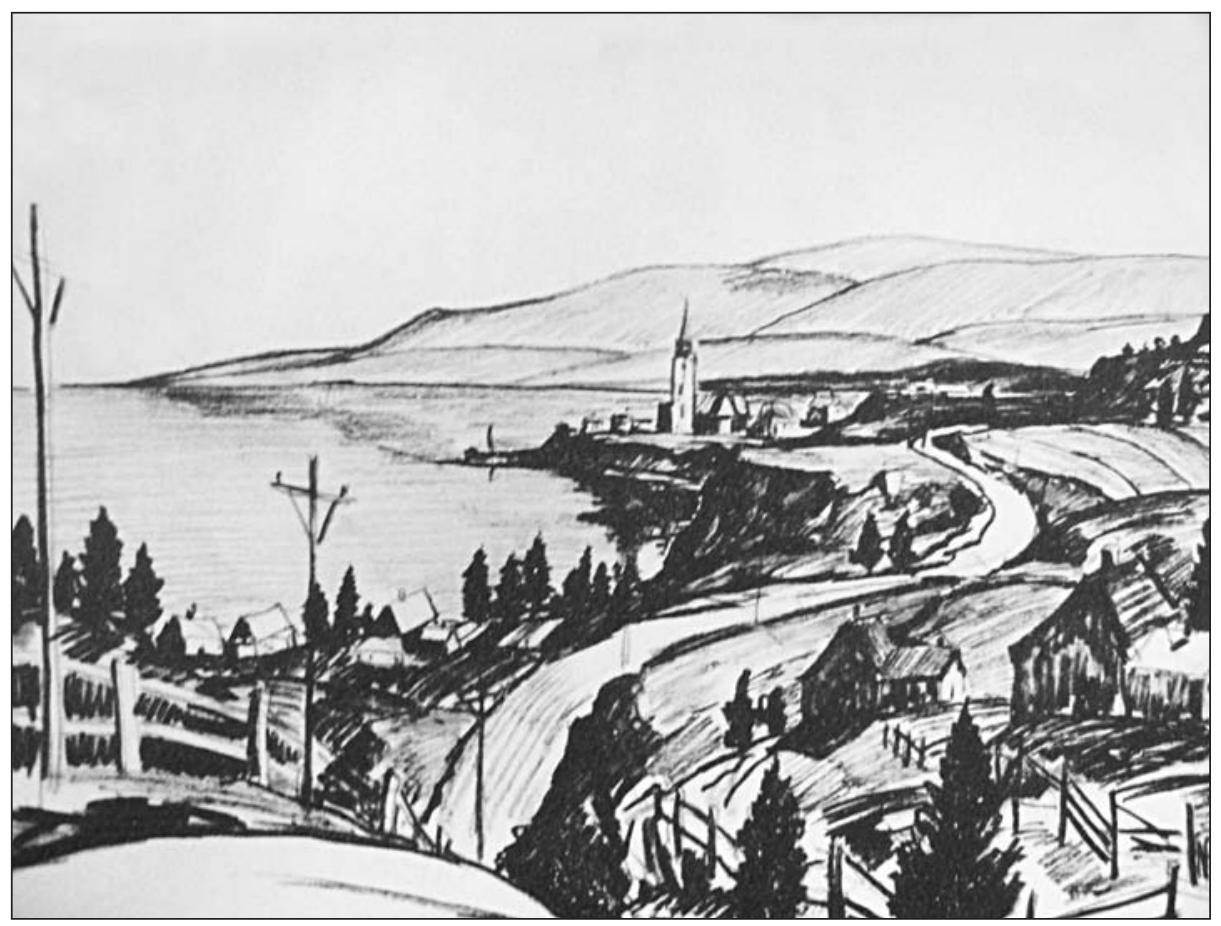

La paroisse de Saint-Paul-des-Capucins en Gaspésie par Putnam Brinley Source: K. G. Brinley, Away to Gaspé (1935).

Une route sinueuse ou difficile sert aussi de prétexte pour présenter les accidents de la géographie. Les récits qui traitent de la Gaspésie sont particulièrement fertiles de ce type de description. Afin d'illustrer le degré considérable de la dénivellation de la route entre Sainte-Anne-des-Monts et Rivière-à-Claude, Brinley mentionne qu'au sommet, une dizaine d'enfants s'amusent à regarder les automobiles monter ${ }^{46}$. Pour les Hogner ou pour les Brinley, l'arrêt en terrain accidenté est aussi une occasion de peindre (lui) et de décrire (elle) le paysage qui s'offre à la contemplation.

D’ailleurs, plusieurs artistes semblent utiliser le voyage en automobile pour peindre, dessiner ou écrire. À la lecture des récits, les liens entre tourisme automobile et l'art semblent étroits, le premier favorisant le deuxième. Les artistes y partagent leur conception de l'art et en quoi les paysages qu'ils croisent les amènent à renouveler leur perspective. Dès

46. Kathrine Gordon Brinley, Away to the Gaspé (New York, Dodd, Mead and Company, 1935), 87. 
son ouverture, la route de la Gaspésie a attiré de nombreux artistes de renom tels que l'Américaine Georgia O’Keeffe. En 1932, celle-ci visite la région et s'en inspire pour peindre une série de tableaux représentant des granges, des croix de chemin et des scènes maritimes.

L'arrêt permet aussi d'observer le mouvement dans un paysage sur une plus longue durée. Kathrine Brinley s'amuse, à partir de sa cabine de Péribonka, à décrire le va-et-vient d'une ferme pendant toute une journée: l'heure de la traite, la sortie et l'entrée des vaches dans l'étable, le foin que les fermiers y amènent et qui se répand sur la route ${ }^{47}$. Ce mouvement qu'elle observe dans le paysage met en évidence l'idée que dans la plupart des descriptions, l'automobile se déplace dans un paysage fixe ${ }^{48}$. L'automobile, par sa vitesse, ne semble pas permettre de saisir les changements ou le mouvement d'un paysage (dans le temps) aussi finement que lors d'un arrêt. Dans les récits étudiés, la description d'un paysage correspond davantage à une succession de tableaux ou de photographies qu'à un film. C'est d'ailleurs précisément sur ce point que la démarche de l'auteur d'un récit rejoint celle du peintre.

On pourrait aussi dire que le paysage du touriste n'est pas nécessairement le même que celui des guides. Ainsi, on ne trouve pratiquement aucune description de voyage en automobile dans le Nord ontarien. À l'inverse, alors que les guides ontariens insistent très peu sur les milieux ruraux, les récits font de la campagne leur paysage privilégié, tant au Québec qu'en Ontario. Les auteurs consacrent plusieurs lignes, voire plusieurs pages, aux types de culture, aux activités des hommes et des bêtes, à l'architecture particulière des bâtiments et des habitations ainsi qu'aux odeurs et aux couleurs qui sont détaillées au point où le lecteur a l'impression d'être sur les lieux. Les milieux ruraux y sont hautement idéalisés. Dans l'article de Frank Yeigh figurent des images des campagnes ontarienne et québécoise qui rappellent les représentations romantiques de l'Arcadie ou des jardins d'Éden ${ }^{49}$. Pour la majorité des auteurs, ces milieux ne sont pas déshumanisés.

\section{Les populations rencontrées sur le territoire touristique}

Au Québec, les descriptions de voyage en automobile sont marquées par la fascination des touristes à observer les habitants dans leurs activités

47. K. G. Brinley, Away to Quebec..., op. cit., 102-103.

48. Ce qui rejoint en quelque sorte les observations de Michel Conan: «Mouvement et métaphore du temps », dans Philippe Poullaouec-Gonidec, Sylvain Paquette et Gérald Domon, dir., Les temps du paysage (Montréal, PUM, 2003), 23-35.

49. F. Yeigh, «The Passing Panorama of a Windshield», loc. cit., 125. 
quotidiennes, des activités qui sont perçues comme étant traditionnelles. Ces touristes américains, ontariens, voire québécois cherchent constamment des yeux les signes d'un mode de vie ancien qui se distingue de leur vie urbaine. Pour un touriste torontois, l'utilisation d'un cheval dans la campagne québécoise est exotique, «[...] an old world complexion ${ }^{50}$ ». Toutefois, si les touristes décrivent dans leurs mots ces hommes et ces femmes occupés aux travaux des champs, une distinction existe par rapport à l'habitant des guides touristiques. Sous la plume des touristes, l'habitant n'est pas un personnage figé dans un tableau: il est en mouvement, il prend vie et sa rencontre balise le récit. Il y a interaction et échange verbal entre le voyageur et l'habitant. Ses coutumes, ses pratiques et ses manières de vivre sont minutieusement étudiées et discutées.

Dans son récit d'un tour de la Gaspésie, l'Ontarienne Margaret Pennell présente le voyage en territoire québécois comme l'occasion d'apprendre à connaître les cousins canadiens-français (c'est le terme qu'elle utilise), d'observer leurs coutumes et surtout d'échanger avec eux, ne serait-ce que quelques mots en dépit de la barrière linguistique ${ }^{51}$. Fascinée par la culture canadienne-française, voire nostalgique du mode de vie des Gaspésiens, coupés qu'ils sont de la société de consommation, Pennell n'affiche jamais une attitude de supériorité culturelle. D’ailleurs, cette admiration partagée par plusieurs touristes envers les Canadiens français, et plus spécifiquement pour la culture de l'habitant, se manifeste dans la majorité des textes étudiés. Elle contribue à façonner une représentation idyllique du mode de vie des populations rurales ou de celles de la péninsule gaspésienne. La mention par les Hogner de l'hospitalité à l'ancienne et du sens du partage d'une famille de la Baie des Chaleurs fait écho à la propagande des guides touristiques qui tend à montrer l'existence d'un mode de vie centré sur l'essentiel et la simplicité.

Les récits étudiés suggèrent que le déplacement en automobile fournit l'occasion de nouer des contacts profonds, de s'attarder plus longuement dans une communauté, d'observer, de discuter, d'analyser les conditions de vie des populations. L'autocamping relève de la même dynamique. En campant chez l'habitant et en mangeant à sa table, les Hogner posent un regard qui semble aller bien au-delà de l'image proposée par la propagande touristique. De manière semblable, ces touristes deviennent «accessibles » à la population alors qu'ils campent pendant quelques jours sur

50. R. A. Gray, «A Motor Trip with a Sketch Box», Canadian Motorist, 17, 4 (avril 1930) : 133-134.

51. Margaret Pennell, "Some observations on a Gaspé Tour Made by Four Girls in a Car, Part 2», Canadian Motorist, 20, 3 (mars 1933): 76. 
une plage. Les résidents du village de Little Gaspé visitent leur campement et s'assoient avec eux pour discuter. Cette proximité permet aux touristes de saisir la réalité sociale et économique de la communautés2.

En revanche, l'importance croissante du tourisme dans les activités économiques des populations rurales ou de celles de la Gaspésie est perceptible dans les témoignages touristiques et contribue à ébranler une vision idyllique de l'habitant ou du pêcheur gaspésien. Ainsi, Jean Middleton Donald et Dorothy Hogner sont frappées de la rapidité avec laquelle les populations québécoises ont compris comment tirer profit du tourisme. Elles ont l'impression que dans certaines localités (Percé, Gaspé et Sainte-Anne de Beaupré), l'économie touristique a supplanté les activités traditionnelles. La commercialisation des abords des routes est une question qui revient souvent dans leur récit. Ces chiens attelés, que plusieurs d'entre eux observent, semblent témoigner d'une marchandisation grandissante du paysage rural québécois ${ }^{53}$. Les Hogner se font continuellement solliciter par des marchands ambulants et dans les villages, enfants et vendeurs de souvenirs font tout pour arrêter leur automobile ${ }^{54}$.

Cette proximité avec les populations locales que permet le tourisme automobile, mais aussi l'activité économique à laquelle il donne lieu, a pour conséquence une certaine remise en question de l'authenticité de la province, telle que publicisée par les guides. Certains touristes ont l'impression de contempler une pièce de théâtre grandeur nature, orchestrée par les populations locales. Les Hogner donnent l'exemple d'une femme filant la laine assise sur une plateforme à proximité de la route. À ses côtés se tient un enfant costumé à l'ancienne; l'ensemble constitue pour les touristes la photographie parfaite: «Several cars stopped and took a snap of this theatrical setup placed there so that no tourist would be disappointed in the "atmosphere". Quaintness with a capital $Q^{55}$.» Ces manœuvres répétées poussent certains touristes à comprendre pourquoi les populations québécoises délaissent leurs occupations traditionnelles pour le dollar touristique. En observant et en discutant avec différentes personnes, les touristes apprennent que les populations de Gaspé et de Percé vivent mieux de l'industrie touristique naissante que de la pêche à la morue.

52. D. C. Hogner, Summer Roads to Gaspé, op. cit., 107.

53. Cette pratique se développe dans les années 1920 et 1930. Afin de faire quelques sous, des enfants attachent un chien devant un petit char (dogcart) et proposent aux touristes de se faire photographier en leur compagnie.

54. J. M. Donald, Quebec Patchwork, op. cit., 164-178.

55. D. C. Hogner, Summer Roads to Gaspé, op. cit., 84. 
Figure 6

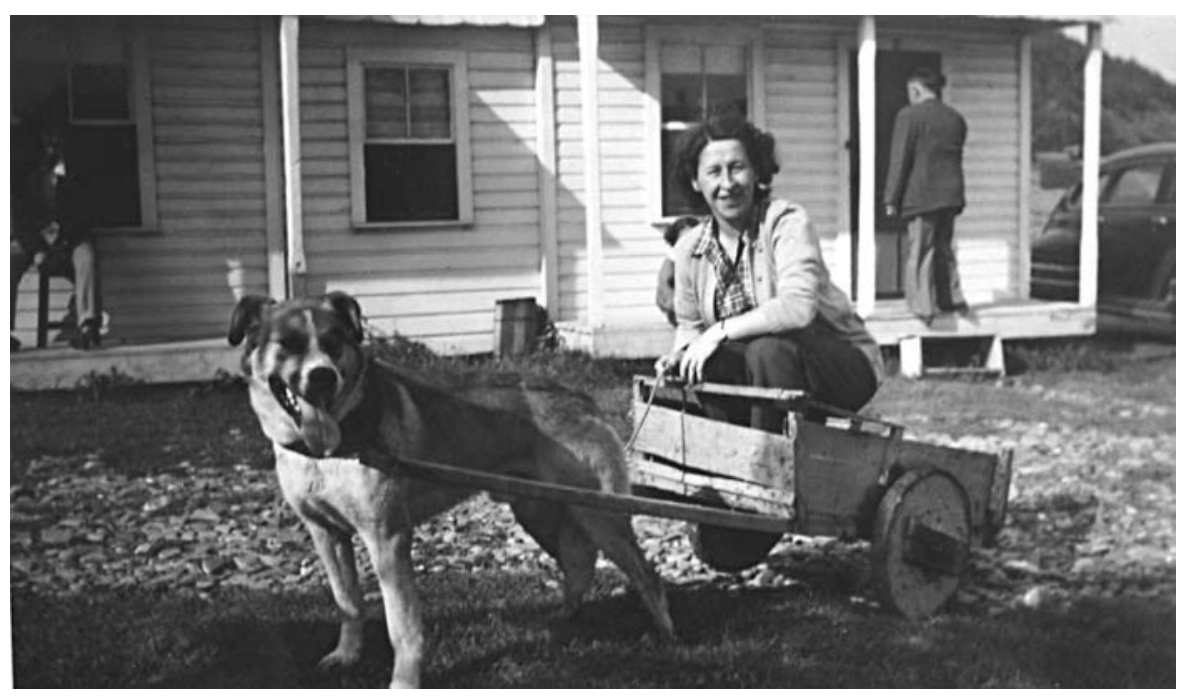

On trouvait ces chiens en attelage un peu partout le long du réseau routier québécois, surtout à proximité de la basilique Sainte-Anne de Beaupré ou sur le parcours gaspésien. Sur cette photographie, ma grand-mère prend la pose lors d'un tour de la Gaspésie en 1949.

Source: Collection de l'auteure.

Les populations locales représentent donc un élément important de l'expérience touristique du territoire québécois. Toutefois, elles doivent se conformer aux rôles que les touristes leur attribuent. Dans un rapport idéal au territoire tel qu'entrevu par les touristes, l'habitant cultive la terre, alors que le pêcheur gaspésien vit de la mer. Ainsi, ni l'un ni l'autre ne doivent s'improviser commerçants ou vendeurs de pacotilles, et ce, au risque d'altérer leur authenticité. La perception des populations locales en Ontario est bien différente de celle au Québec. Absentes des guides ontariens, celles-ci le sont tout autant des récits sur l'Ontario. Tout au plus, des échanges ont lieu dans les hôtels, les stations d'essence ou les bureaux de renseignements touristiques.

\section{CONCLUSION}

L'approche d'histoire environnementale privilégiée dans cet article a permis de montrer que les territoires touristiques n'existent pas d'emblée et qu'ils sont le produit de différents regards, pratiques et gestes posés sur le territoire. Dans ce cas-ci, la technologie automobile oriente profondément le développement touristique. On a pu voir comment les acteurs 
du tourisme ont récupéré et adapté des conceptions anciennes et même stéréotypées de l'environnement québécois et ontarien. Alors que l'Ontario continue de se présenter comme un territoire romantique et en marge de la civilisation, le Québec est montré comme un lieu où subsistent, à travers le mode de vie de sa population, les traces d'une culture française et catholique. Le recours à la peinture ou à la gravure pour composer les représentations en couverture des guides témoigne de l'antimodernité qu'inspirent ces deux territoires, en mettant en avant leur caractère ancien et leur pureté. L'art contribue à construire et à entretenir des mythes, voire à édifier certains paysages emblématiques censés représenter l'ensemble de la province.

La littérature, les recherches dans le domaine de l'histoire et de l'ethnologie ainsi que les premiers efforts dans la mise en valeur et la protection du patrimoine fournissent aussi aux acteurs du tourisme les éléments permettant de composer et d'actualiser ces représentations du Québec et de l'Ontario. Dans tous les cas, la promotion touristique met en scène le mouvement de l'automobile et l'accessibilité des territoires touristiques. Les guides ontariens insistent sur les milieux forestiers et aquatiques, liés à l'idée d'un territoire sauvage et récréatif. Présenté comme un immense terrain de jeux, l'Ontario étend continuellement ses dimensions, repoussant toujours plus au Nord la limite du territoire touristique. Le Québec, quant à lui, tout en continuant à insister sur des régions touristiques bien établies, s'ouvre au tourisme motorisé dans l'Est (Gaspésie). Si l’historiographie a surtout insisté sur la dimension culturelle de la promotion touristique, une analyse iconographique des guides montre l'importance qu'y tient la mise en scène des milieux ruraux. On peut y distinguer l'image d'une campagne hybride, où l'environnement bâti côtoie des éléments naturels altérés ou modifiés par l'homme. Cela illustre que les frontières entre environnement naturel et bâti ne sont pas nettes, mais plutôt mouvantes, d'où la nécessité d'élargir la notion d'environnement et d'y inclure tant l'espace, le territoire, les milieux biogéophysiques, les animaux, les plantes que l'être humain.

De leur côté, les récits touristiques permettent de saisir les différentes manières par lesquelles les touristes participent à la création de ces représentations du territoire touristique, mais aussi à son appropriation. En qualifiant ce territoire et en décrivant les éléments qui le constituent (et qui attirent son attention), le touriste compose de nouvelles représentations. La qualification du territoire semble varier en fonction des clientèles. Ainsi, les Américains voient dans l'Ontario et le Québec des terri- 
toires attirants pour leur climat frais, favorisant l'expérience de la solitude et offrant la possibilité de découvrir des milieux naturels et habités authentiques. Pour les Canadiens, et plus précisément les Québécois et les Ontariens, la nostalgie semble être la motivation au cœur de leur voyage.

Le paysage de l'automobiliste est une autre manière de lire le territoire touristique. Comme la photographie, le touriste décrit les éléments qui se succèdent sur sa route et qui composent le paysage. La description du roulement de l'automobile cherche à donner l'impression au lecteur d'être à bord, de s'imaginer en train de traverser ce paysage, voire de se l'approprier à son tour. La turbulence du véhicule permet d'aborder les caprices de la géographie: une montée particulièrement difficile rend compte d'une dénivellation importante, d'un paysage montagneux. De nombreux touristes automobilistes se révèlent également des artistes et le regard qu'ils portent à partir de leur automobile leur permet de renouveler leurs perspectives.

L'immobilité de l'habitant est un autre de ces aspects sur lequel les récits divergent des guides touristiques. Sous la plume des voyageurs, les populations rurales sont mouvantes, ouvertes aux échanges et même aux confidences. Les touristes saisissent l'occasion de ce rapprochement, mais celui-ci révèle des conditions de vie difficiles et une pauvreté qui s'incarne à travers des enfants qui quêtent le long des routes. Conséquemment, les touristes portent un regard de plus en plus critique sur la figure de l'habitant (ou du pêcheur gaspésien), mais sans pour autant remettre en question sa véracité. L'importance de leur sentiment antimoderniste ne leur permet peut-être pas d'endosser un discours qui dénoncerait ces conditions de vie. Les efforts déployés par les gouvernements pour déconstruire ces représentations après 1945 et les remplacer par l'image d'un Ontario au patrimoine culturel riche et d'un Québec urbain et moderne (mais qui se distingue toujours par son caractère français) montrent bien la construction culturelle dont les territoires touristiques font l'objet. 